Rumination and Impaired Prospective Memory

Authors:

K. Fredman Stein

W.L. Morys-Carter

L. Hinkley 


\begin{abstract}
Prospective memory (PM), or remembering to remember, is crucial to everyday functioning. Understanding factors associated with PM impairments is thus important. One likely factor, not investigated to date, is rumination: a common cognitive process comprising repetitive self-focused thoughts. We investigated whether rumination (both state and trait) is associated with impaired PM, and whether any associated impairment is exacerbated in the context of negative stimuli. A sentence-rating task with sentences varying in valence was used with embedded PM cues in a non-clinical sample $(N=60)$. Both PM cue detection and response times to PM cues were measured. State rumination (measured by ruminative self-focus), two trait rumination subtypes (reflective pondering and brooding) and mood were assessed. Results showed that state rumination was associated with impaired PM cue detection across the valences. State rumination was also associated with slower response times to PM cues embedded in negative sentences (but not in positive or neutral sentences). Trait brooding was associated with an overall slowed response times to PM cues (although reflective pondering was not), consistent with literature suggesting that brooding is the more maladaptive rumination subtype (De Lissnyder et al., 2010; Joormann, Dkane, \& Gotlib, 2006). These findings suggest that trait and state rumination are associated with dissociable PM impairments: while state rumination is associated with a greater impairment in PM (missing PM cues), trait rumination is associated with an overall reduction in the speed of PM responses. Furthermore, the finding that state (but not trait) rumination was related to slower PM response times only in a negative context, suggests it is state rumination specifically that increases the time taken to disengage from negative material. However, further investigation is needed to establish causality.
\end{abstract}


Key words: rumination; prospective memory; negative valence; brooding 


\section{Rumination and Impaired Prospective Memory}

Prospective memory (PM), the process of remembering to remember, plays a crucial role in everyday functioning and achieving goals. Persistent PM failures can have wide-ranging adverse consequences (for example where remembering to take medication is regularly forgotten), and so it is important to understand which factors may be associated with impairments in PM.

One factor which could be associated with impaired PM performance and that to-date has not been investigated, is rumination, a process comprising repetitive and persistent selffocused thoughts (Nolen-Hoeksema, 2000). There is evidence to suggest that people differ in their tendency to ruminate (Nolen-Hoeksema, 1991). This tendency has been found to display considerable stability over time, and so can be regarded as a trait (Roberts, Gilboa, and Gotlib, 1998; Nolen-Hoeksema, 2000). This has been distinguished from state rumination, which is a measure of whether a person is currently ruminating. Two subtypes of trait rumination have been identified: reflective pondering, a form of 'cognitive problem solving' in response to low mood; and the more maladaptive brooding, which can be defined as 'a passive comparison of one's current situation with some unachieved standard' (Treynor, Gonzalez, \& Nolen-Hoeksema, 2003, p.256).

Critically, rumination is a core cognitive process in depression (Nolen-Hoeksema, 2000) and depression has already been shown to impair PM. In a time-based PM task, Rude, Hertel, Jarrold, Covich and Hedlund (1999) found that depressed participants scored significantly lower and monitored the time less than controls. Given that depression is a multicomponent disorder, it is important to understand which aspects of depression may be associated with impairments in PM. As well as rumination, low mood is a key component of 
depression. Interestingly, research into the relationship between mood and PM is largely inconclusive. Kliegel et al. (2005) found that induced sad mood resulted in poorer PM performance, although the effect was significant only in the first half of the task. Similarly, Knight, Brewer, Ball, DeWitt, and Marsh (2015) found only weak evidence for a relationship between sad mood and poorer PM performance. On the other hand, Rummel, Hepp, Klein, and Silberleitner (2012) found that PM performance was actually improved in an induced sad mood compared to happy or neutral. This inconclusive evidence in relation to the effects of low mood on PM suggests that other aspects of depression which are likely to impair PM need to be investigated.

Although not previously investigated, considerable evidence suggests that rumination may be the specific component of depression which affects PM. Altgassen, Kliegel and Martin (2009) found that individuals with depression showed impaired PM performance compared to controls when PM cues were not directly related to the on-going task (non-focal cues). However, when cues were directly related to the on-going task (focal cues), no significant difference was found. As focal cues have been found to be retrieved more automatically than non-focal cues (McDaniel \& Einstein, 2000), this suggests a selective deficit in tasks that place higher demands on monitoring. While the reason for this has not been established, one plausible explanation is that it may be due to the competing attentional demands of rumination which has been associated with depression.

There is substantial evidence to suggest that rumination occupies the attentional resources available for task-relevant processing. Both state and trait rumination have been associated with impairments in several cognitive processes (e.g. Davis \& Nolen-Hoeksema, 2000; De Lissnyder, Koster \& De Raedt, 2011; Watkins \& Brown, 2002; Whitmer \& Banich, 2007). For example, in a random number generation task, Watkins and Brown (2002) found that induced state rumination was related to impaired ability to produce random numbers. 
This indicates that ruminative thoughts may consume resources involved in inhibiting prepotent responses (in this case counting in order). Furthermore, using a task switching paradigm with dysphoric and non-dysphoric participants, De Lissnyder et al. (2010) found that high trait ruminators showed impairments in set-shifting (a form of cognitive flexibility which involves switching attention between tasks) and in inhibiting negative material. Brooding was most strongly predictive of both impaired inhibition and set-shifting, a finding which supports previous research (Whitmer \& Banich, 2007). In contrast, depressive symptoms were not associated with impaired inhibition or set-shifting. This suggests that rumination, particularly brooding, may be more proximally linked to impairments in inhibition and set-shifting than depressive symptoms in general (De Lissnyder et al., 2010).

Findings that rumination has been associated with impaired inhibition and set-shifting (De Lissnyder et al. 2010; Whitmer \& Banich, 2007) may be relevant in a PM context. Inhibition plays an important role in PM, both during intention initiation (identifying the appropriate time to perform an action), and intention execution (performing the intended action; Kliegel Jäger, Altgassen, \& Shum, 2008). Set-shifting is also important for PM, which requires switching attention between on-going tasks and retrieval of the prospective intention (Kliegel et al., 2008). Thus, it is plausible that rumination may impair PM performance, due to its competing demands on attentional resources.

A second reason why rumination may affect PM is due to the effects of negatively valenced stimuli on both PM and rumination. Across two event-based PM tasks, Clark-Foos, Brewer, Marsh, Meeks and Cook (2009) found that PM performance was impaired when either PM cues or PM context were negatively valenced compared to when they were positively or neutrally valenced. The authors suggest that valenced stimuli, particularly negative stimuli, evoke more task-irrelevant associations than neutral material, and so serve as distractors which impair PM processes. 
It is possible that this adverse effect of negatively valenced stimuli on PM in general (Clark-Foos et al., 2009) may be particularly pronounced in rumination, given evidence suggesting that state and trait rumination are related to difficulties in inhibiting, or disengaging from negative material (Joormann \&Vanderlind, 2014; Koster, De Lissnyder, Derakshan, \& De Raedt, 2011). For example, De Lissnyder et al. (2011) found that rumination was related to impairments in switching when negative material is held in working memory, a memory system found to be closely associated with the prospective component of PM (Arnold, Bayen \& Smith, 2011). In this study, participants were required to keep separate mental counts of the number of neutral versus angry faces (the emotional condition) and male versus female faces (the non-emotional condition). Switch costs were measured by the difference in response time between trials in which participants were required to switch (e.g. from angry to neutral), and trials in which no switching (e.g. neutral to neutral) was required. In the non-emotional condition, there was no effect of rumination. However, in the emotional condition, high rumination was associated with greater switch costs when switching from angry to neutral counts. Thus, the adverse effects of negative stimuli on PM found by Clark-Foos et al. (2009) may be exacerbated in state and trait rumination due to increased time needed to inhibit or disengage from negative material.

The present study aimed to examine whether rumination is related to impaired PM in a valenced context. To our knowledge, this question has not previously been investigated. A valenced sentence-rating task developed by Clark-Foos et al. (2009) was used. Given evidence indicating that even high trait ruminators experience substantial fluctuation in the extent of their state rumination (Moberly \& Watkins, 2008), both state and trait rumination were examined. In order to investigate the effects of rumination without the influence of depression, individuals with depression were excluded in order to control for this potential confound. Nonetheless, there is substantial evidence for considerable variability in 
rumination within non-depressed samples (for example, Moberly \& Watkins, 2008). As low mood is associated with rumination (Moberly \& Watkins, 2008; Nolen-Hoeksema, Wisco, \& Lyubomirsky, 2008), and there is some evidence suggesting that mood may affect PM performance (Kliegal et al., 2005; Knight, 2015; Rummel et al., 2012; Schnitzspahn et al., 2014), a mood scale was also used in order to disentangle the respective effects of mood and rumination. Both cue detection and response times to PM cues were measured, in order to capture changes in different aspects of PM; while impaired response times would indicate a slowed remembering, impaired cue detection would indicate an increase in complete forgetting.

The study examined two hypotheses.

Hypothesis 1: We hypothesise that both state and trait rumination would be related to impairments in PM performance across statements varying in valence. This is based on evidence suggesting that PM often requires attentional resources, and that both state and trait rumination are associated with depleted attentional capacity. Given that inhibition and setshifting are needed for PM, and rumination has been associated with impaired inhibition and slowed response times when set-shifting (De Lissnyder et al. 2010; Whitmer \& Banich, 2007), we extend this hypothesis to both PM cue detection and response times to PM cues.

Hypothesis 2: We hypothesise that any impairment of response times to PM cues associated with state or trait rumination would be exacerbated in the context of negatively valenced sentences. This hypothesis is based on evidence which indicates (1) an adverse effect of negative valence on PM performance, and (2) an association between rumination and slower response times when required to inhibit or disengage from negative material.

\section{Method}




\section{Participants}

Sixty-six participants were recruited from a University using posters, block emails and one researcher distributing information sheets at the University entrance. Participants were required to speak fluent English, be aged between 18-60 years, and not have a diagnosis of clinical depression. No incentives were given for participation. Thirty-three participants were randomly assigned to each of two PM target conditions (food and vehicles). Four participants were excluded because they failed the retrospective memory (RM) check (see below). Two participants with missing data were excluded, leaving a final sample of 60 participants: 38 women, 21 men, and one with a non-binary gender (age range $=19-55$ years, $M=28.7, S D=$ 8.3). This sample comprised a mix of students and staff working at the University.

\section{Measures}

Ruminative Responses Scales (RRS; Nolen-Hoeksema \& Morrow, 1991). The RRS is a 22-item self-report measure of trait tendency to ruminate. It asks participants to identify how often they ruminate in response to low mood on a 4-point scale, ranging from almost never (1) to almost always (4). Higher scores indicate a greater trait tendency to ruminate. Items describe responses to low mood 'that are self-focused...symptom focused... and focused on the possible consequences and causes of the mood' (NolenHoeksema, Larson, \& Grayson, 1999, p.1064). As some items on the RRS are confounded with depression content, Treynor et al. (2003) conducted a factor analysis to identify distinct subtypes which exclude those items. They identified two psychometrically valid subtypes which differentially relate to depression in terms of predictive ability: reflective pondering, comprised of five items designed to assess the extent to which 'cognitive problem solving' is used in response to low mood, and brooding, comprised of five items which assess the extent 
to which individuals engage in 'a passive comparison of one's current situation with some unachieved standard' (Treynor et al., 2003, p.256). In order to test rumination in a way which is not confounded by depressive content, these two subtypes were used for analysis in this study. In our study, Cronbach's alpha coefficients were .75 for reflective pondering, and .70 for brooding. In a randomised community sample of adults in the US $(N=1130)$, Treynor et al. (2003) reported norms for both the brooding $(M=9.83, S D=3.11)$, and reflective pondering $(M=9.40, S D=2.96)$ subscales.

Ruminative self-focus (adapted from Moberly \& Watkins, 2008). This composite measure comprises two bipolar nine-point scales, which ask participants to rate the extent to which their thoughts have been 1) focused on their feelings, and 2) focused on their problems, in the last few hours. Higher scores indicate greater ruminative self-focus. A composite ruminative self-focus score is calculated by summing the $\mathrm{z}$ scores of these two scales (Moberly \& Watkins, 2008; $\alpha=.59$ ).

Mood scale (Roberts, Watkins \& Wills, 2013). A visual analogue mood scale was also included in order to control for the effects of mood. Participants were asked to rate how happy or sad they had been feeling in the last few hours on a nine-point scale, ranging from 0 (very sad) to 9 (very happy). Scales of this kind have been shown to provide sensitive and reliable measures of mood (e.g. Watkins \& Teasdale, 2001).

Distractor task. Participants were asked to complete three minutes of basic arithmetic questions. This distractor task was used between the instructions for the PM task and the PM task itself in order to prevent PM rehearsal or the formation of PM strategies (Clark-Foos et al., 2009).

Sentence-rating and PM task (adapted from Clark-Foos et al., 2009). For the sentence-rating task participants were asked to rate the valence of 90 sentences selected from 
the large Maratos and Rugg (2001) corpus on a seven-point scale: 30 positive $(M=6.50), 30$ neutral $(M=5.00)$ and 30 negative $(M=2.87)$. For the PM task, six neutral PM cues were embedded within six valenced sentences (two of each valence). Participants were randomly assigned to one of two PM target conditions (vehicles or food) and asked to respond to cue sentences containing their target category (by pressing the ' $t$ ' key). For example, participants assigned to the food condition were required to press the ' $t$ ' key before making their valence rating for sentences containing a food item, such as 'Those who had eaten oyster got chronic diarrhoea' (negative) 'She put the rice on to boil and set the time for 20 minutes' (neutral) 'The baking bread smelled delicious' (positive). Cue sentences were matched for valence across the two conditions with sentence order randomised by the software in order to control for priming effects.

\section{Design}

This study employed a mixed design, with five independent variables (IVs) and two dependent variables (DVs). The repeated-measures IV was sentence valence, with three levels: positive, negative and neutral. The between-group IVs were group (food and vehicles) trait brooding and trait reflective pondering (measured by RRS subscales), and state rumination (measured by ruminative self-focus scales). The DVs were cue detection accuracy (proportion of targets spotted) and response times to PM cues (measured in milliseconds). Mood (measured by a mood scale) was also included as a covariate to control any confounding effects. Two PM target groups (vehicles and food) were used to ensure that the results would be generalizable beyond one particular PM target category (Clark-Foos et al., 2009). In order to control for potential confounding effects of age-related memory decline and depression, only participants under 60 years without a diagnosis of depression were included in the sample. 


\section{Procedure}

After providing informed consent, participants were asked to complete the RRS, ruminative self-focus, and mood scales. The sentence-rating task and PM task were then presented on a PC using PsychoPy (Peirce, 2007). Onscreen instructions asked participants to rate each sentence on a scale of 1-7 (with 1 being very negative, 7 very positive, and 4 neutral) and to press the ' $t$ ' key on their keyboard when they saw either a vehicle or food item (depending on their PM target condition) before making the sentence rating judgement. They were asked to proceed as quickly and accurately as possible. Participants were asked to repeat the instructions back to the researcher to confirm their understanding. Participants then completed the three-minute arithmetic distractor task, before proceeding with the sentence rating and embedded PM tasks (Clark-Foos et al., 2009). At the end of the study, the experimenter asked the participants to repeat the instructions again, in order to ensure that any errors were due to PM rather than RM lapses. This was followed by a debrief in which the aims and potential implications of the research were summarised. Participants were also given signposting information to relevant support services if they felt that completing the rumination measures was upsetting. The research was approved by the Oxford Brookes University Psychology Research Ethics Committee. 


\section{Results}

Rumination and mood scores are presented in Table 1. In order to check that the sentences were perceived as sufficiently different in terms of their valence, a repeated-measures ANOVA was conducted on participants' mean sentence ratings. This showed that valence (neutral $M=4.25$, positive $M=6.05$, negative $M=1.85$ ) had a highly significant effect on sentence rating, $F(2,122)=656.69, p<.001, \eta_{p}^{2}=.92$. Post hoc tests revealed that positive sentences were rated significantly more highly than neutral sentences, which in turn were rated significantly more highly than negative sentences (all at $\mathrm{p}<.001$ with Bonferroni corrections).

ANCOVAs were conducted on each DV (proportion of cues detected and response times to PM cues) with valence and group as IVs. State rumination, trait rumination measures and mood were included as covariates in order to keep these measures as continuous scales. Covariates were checked for multicollinearity, and as none of the correlations between the covariates exceeded .40, all could be included in the analysis (Field, 2013).

In order to investigate the valence-specific effects of trait and state rumination on PM cue detection, a mixed design ANCOVA was conducted with group and valence as IVs, and with four covariates; the two trait rumination subscales (brooding and reflective pondering), ruminative self-focus and mood. Mauchly's test indicated that the assumption of sphericity was not violated, $\chi^{2}(2)=3.64, p=.162$. Shapiro-Wilk tests showed significant deviations from normality in the residuals, but since transformations were unable to improve their distributions analysis was performed on the original proportions and the results for this ANCOVA were treated as tentative. No significant effect was found of valence on PM cue detection, $F(2,53)=1.80, p=.176, \eta_{p}^{2}=.06$. Of the four covariates, only ruminative selffocus showed a significant main effect on proportion of cues detected, $F(1,54)=6.11, p=$ 
$.017, \eta_{p}^{2}=.10$, with those with greater ruminative self-focus scores detecting fewer PM cues $(r=-.29)$.

The only significant interaction in this analysis was between valence and mood, $F(2$, $108)=3.87, p=.024, \eta_{p}^{2}=.07$. In order to investigate this interaction further, Spearman's rho correlations were conducted between mood and cue detection in sentences of each valence (see Table 3), and Quantpsy software was used to calculate the difference between the correlations (Lee \& Preacher, 2013). The correlations between cue detection and mood were significantly different for negative and neutral sentences $(z=1.98, p=.047)$. This indicates that individuals scoring higher on the mood scale detected more cues embedded in negative sentences than those scoring lower, compared to neutral sentences.

In order to investigate valence-specific effects of trait and state rumination on response times to PM cues, a repeated-measures ANCOVA was conducted with group and valence as IVs, and with four covariates; the two trait rumination subscales (brooding and reflective pondering), ruminative self-focus and mood. The residuals in the initial ANCOVA significantly deviated from normality but this deviation was eliminated in the negative valence condition and reduced in the neutral and positive conditions by performing the ANCOVA on log transformed response times instead. Mauchly's test indicated that the assumption of sphericity was not violated, $\chi^{2}(2)=.41, p=.815$. The results show that there was no significant main effect of valence on response times to PM cues, $F(2,46)=2.71, p=$ $.077, \eta_{p}^{2}=.11$. The only covariate to show a significant main effect was trait brooding, $F(1$, $47)=10.08, p=.003, \eta_{p}^{2}=.18$, where those with higher brooding scores took longer to respond to PM cues $(r=.25)$. 
There was a significant interaction between ruminative self-focus and valence, $F(2,46)=$ $7.28, p<.002, \eta_{p}^{2}=.24$. In order to investigate this interaction further, Pearson's correlations were conducted (see Table 4). These indicated a significant association between greater ruminative self-focus and longer response times to cues embedded in negative sentences $(r=$ $.32, p=.018)$. Ruminative self-focus did not significantly correlate with response times to PM cues in neutral or positive sentences. There was also a significant interaction between valence and group, $F(2,46)=5.87, p=.005, \eta_{p}^{2}=.20$. Pairwise comparisons of the three levels of valence within each group showed that the response times to PM cues in negatively valenced food sentences were slower than neutral and positive, whereas there was no effect of valence for the vehicle sentences.

\section{Discussion}

The present study aimed to examine whether rumination is related to impairments in PM performance, and if so, whether these impairments are exacerbated in the context of negatively valenced material. We found that (1) state rumination was associated with impairments in PM cue detection, and with longer response times to PM cues embedded in negative (but not positive or neutral) sentences (2) trait brooding was related to longer response times to PM cues, but not to impaired PM cue detection (3) trait reflective pondering was not related to either measure of PM performance.

The first hypothesis was that state and trait rumination would be related to impairments in both measures of PM performance. This was partially supported: the relationship between rumination and PM performance was different both for type of rumination, and for PM accuracy and speed. State rumination was found to be related to impairments in PM cue detection, with a medium effect size $\left(\eta_{p}^{2}=.10\right.$; Watson, 2016). In relation to trait 
rumination, individuals with higher scores on the brooding subscale tended to have longer response times to PM cues, with a large effect size $\left(\eta_{p}^{2}=.18\right.$; Watson, 2016). By contrast, reflective pondering was not found to be related to either accuracy or speed. This is consistent with previous research which has found that brooding is the type of trait rumination most closely associated with impairments in cognitive processes (De Lissnyder et al., 2010; Whitmer \& Banich, 2007) and suggests that brooding is more maladaptive than reflective pondering (Joormann, Dkane, \& Gotlib, 2006). This finding may have important implications, as brooding is also the form of rumination which has been most closely linked to depression (Joormann, Dkane, \& Gotlib, 2006). Although not directly investigated by the present study, it may be that brooding plays a mediating role in the relationship between depression and impaired PM performance.

These findings are consistent with previous research which has shown that state and trait rumination are related to impairments in working memory, inhibition and set-shifting (De Lissnyder et al., 2010; De Lissnyder et al., 2011; Watkins \& Brown, 2002). While all these cognitive processes are involved in PM (Arnold et al., 2015; Kliegel et al., 2008), to our knowledge this is the first study to show a specific relationship between state rumination and impaired PM cue detection, and trait brooding and impaired response times to PM cues.

One explanation for these findings is that rumination depletes the attentional resources required by PM processes. This suggests a potential link between two bodies of evidence: one indicating that PM processes require attentional resources in at least some cases $(\mathrm{McDaniel} \&$ Einstein, 2000); and the other indicating that rumination depletes attentional resources (Hertel, 1998, 2004; Levens et al., 2009; Philippot \& Brutoux, 2008; Watkins \& Brown, 2002). However, given that this study was essentially correlational, further investigation is needed to establish causality. 
However, while state rumination was associated with impaired cue detection, it was not associated with impaired response times to PM cues. Furthermore, while trait brooding was associated with impaired response times, it was not related to impairments in cue detection. These findings suggest that trait and state rumination are associated with dissociable PM impairments which may be explained by the differing nature of trait and state rumination. Trait rumination is the tendency to habitually ruminate, which has been shown to interfere with the speed of cognitive processing. State rumination is a specific measure of high current focus on one's own problems and feelings, and so likely to specifically interfere more directly with the task in hand, in this case, remembering to remember.

The present study's findings also provide some support for the study's second hypothesis: that there would be a specific association of state and trait rumination with impaired response times to PM cues in a negatively valenced context. We found that individuals with higher ruminative self-focus scores took longer to respond to cues embedded in negative sentences (but not positive or neutral sentences) than those with lower scores, with a large effect size $\left(\eta_{p}^{2}=.24\right.$; Watson, 2016). This is consistent with De Lissnyder et al.'s (2011) finding of an association between rumination and longer switch costs when shifting attention away from negative stimuli, and so provides support for models proposing that rumination is associated with specific difficulties inhibiting negative material (e.g. Joormann \& Vanderlind, 2014). It is plausible that state rumination impaired individuals' ability to inhibit interference from irrelevant content in the negative sentences, thus slowing retrieval of the PM intention. However, further research is needed to establish whether the relationship is causal. It is interesting that there was no interaction between sentence valence and either measure of trait rumination. This suggests that it is current focus on feelings and problems, rather than a general tendency to ruminate, that increases the time taken to disengage from negative material in a PM context. 
There was also a significant interaction between valence and group. Response times to PM cues in negatively valenced food sentences were slower than response times to PM cues in neutral and positive sentences, whereas there was no effect of valence for the vehicle sentences. One plausible explanation is that as the negative food items referred to food contamination, this may have had a distracting disgust effect that was not present in the negative vehicles sentences.

It is notable that the findings in relation to state rumination and trait brooding were found independently of mood. Given that there was no main effect of mood, and that previous research into the effects of mood on PM is weak and inconsistent (Kliegel, 2005; Knight, 2015; Rummel et al., 2012), it is plausible that rumination plays a more important role in the relationship between depression and impaired PM performance than low mood. To more directly investigate the potential relative mediating roles of rumination and mood in the relationship between depression and impaired PM performance, future research should utilize a sample with clinical depression.

The strengths of the present study include firstly, using established measures of both state and trait rumination, as well as controlling for mood. Secondly, two PM target conditions were used to ensure the results are generalizable beyond a particular category. Thirdly, any participants with RM lapses were identified through checks and excluded to ensure that the task measured PM rather than RM. However, this study had several limitations. Firstly, we used a volunteer sample consisting mainly of students, which raises issues of generalizability. Secondly, the PM tasks undertaken were computer-based experimental tasks, and it remains to be seen whether these results would transfer to real-world situations. Thirdly, although the study excluded individuals with self-reported depression (and controlled for mood), a formal depression screening tool for was not used. Lastly, we were unable to correct for the skew in 
the PM cue detection data, meaning that the PM cue detection results should be taken tentatively. However, it is notable that these findings were in the same direction as the response time results, thus adding support to our hypothesis that state and trait rumination would be associated with impairments in PM performance.

To our knowledge, this is the first study to find evidence of a relationship between (1) state rumination and impaired PM cue detection (2) trait brooding and impaired response times to PM cues and (3) state rumination and longer response times to PM cues embedded in negative sentences. If further research established these relationships in a clinical sample, this could be of therapeutic importance. PM tasks are ubiquitous in everyday life, and are involved in future planning and goal achievement. As state rumination and trait brooding are key features of depression (Nolen-Hoeksema, 2000; Joormann et al., 2006), the finding that they are associated with impaired PM performance could be used to inform interventions aiming to improve PM tasks (such as medication adherence) in depression, as well as chronic conditions where depression is often comorbid, such as hypertension, diabetes and HIV.

\section{References}

Altgassen, M., Kliegel, M., \& Martin, M. (2009). Event-based prospective memory in depression: The impact of cue focality. Cognition \& Emotion, 23, 1041-1055. doi: $10.1080 / 02699930802284158$ 
Arnold, N. R., Bayen, U. J., \& Smith, R. E. (2015). Hierarchical multinomial modeling approaches: An application to prospective memory and working memory. Experimental Psychology, 62, 143-152. doi:10.1027/1618-3169/a000287

Clark-Foos, A., Brewer. G., A., Marsh, R., L., Thadeus Meeks, J., \& Cook, G., I. (2009). The valence of event-based prospective memory cues or the context in which they occur affects their detection. The American Journal of Psychology, 122 (1), 89-97.

Davis, R. N., \& Nolen-Hoeksema, S. (2000). Cognitive inflexibility among ruminators and nonruminators. Cognitive Therapy and Research, 24, 699-711. doi:10.1023/A:1005591412406

De Lissnyder, E., Koster, E. H., Derakshan, N., \& De Raedt, R. (2010). The association between depressive symptoms and executive control impairments in response to emotional and non-emotional information. Cognition and Emotion, 24, 264-280. doi: $10.1080 / 02699930903378354$.

De Lissnyder, E., Koster, E., \& Raedt, R. (2011). Emotional interference in working memory is related to rumination. Cognitive Therapy \& Research, 36, 348-357. doi:10.1007/s10608-011-9352-4

Field, A. (2013). Discovering statistics using IBM SPSS Statistics (4th ed.). London: Sage.

Hertel, P. T. (1998). Relation between rumination and impaired memory in dysphoric moods. Journal of Abnormal Psychology, 107, 166-172. doi:10.1037/0021-843X.107.1.166

Hertel, P. T. (2004). Memory for emotional and non-emotional events in depression: A question of habit. In D. Reisberg \& P. Hertel (Eds.), Memory and Emotion (pp. 186216). New York, NY: Oxford University Press. 
Joormann, J., Dkane, M., \& Gotlib, I. H. (2006). Adaptive and maladaptive components of rumination? Diagnostic specificity and relation to depressive biases. Behavior Therapy, 37, 269-280. doi:10.1016/j.beth.2006.01.002

Joormann, J., \& Vanderlind, W. M. (2014). Emotion regulation in depression. The role of biased cognition and reduced cognitive control. Clinical Psychological Science, 2, 402-421. doi:10.1177/2167702614536163

Kliegel, M., Jäger, T., Altgassen, M., \& Shum, D. (2008). Clinical neuropsychology of prospective memory. In M. Kliegel, M. McDaniel \& G. Einstein (Eds.) Prospective Memory: Cognitive, Neuroscience, Developmental and Applied Perspectives (pp.283302). Taylor \& Francis Group: New York.

Kliegel, M., Jäger, T., Phillips, L.H., Federspiel, E., Imfeld, A., Keller, M., \& Zimprich, D. (2005). Effects of sad mood on time-based prospective memory. Cognition \& Emotion, 19, 1119-1213. doi: 10.1080/02699930500233820

Knight, J. B., Brewer, G. A., Ball, B. H., DeWitt, M. R., \& Marsh, R. L. (2015). The influence of mood on the process and content of encoding future intentions. The Quarterly Journal of Experimental Psychology. doi:10.1080/17470218.2014.975729

Koster, E. H., De Lissnyder, E., Derakshan, N., \& De Raedt, R (2011). Understanding depressive rumination from a cognitive science perspective: The impaired disengagement hypothesis. Clinical Psychology Review, 31, 138-145. doi:10.1016/j.cpr.2010.08.005 
Lee, I. A., \& Preacher, K. J. (2013). Calculation for the test of the difference between two dependent correlations with one variable in common [Computer software]. Available from http://quantpsy.org.

Levens, S. M., Muhtadie, L., \& Gotlib, I. H. (2009). Impaired resource allocation and rumination in depression. Journal of Abnormal Psychology, 118, 757-766. doi:10.1037/a0017206

McDaniel, M. A., \& Einstein, G. O. (2000). Strategic and automatic processes in prospective memory retrieval: A multiprocess framework. Applied Cognitive Psychology, 14, 127144. doi: 10.1002/acp.775.

Maratos, E. J., \& Rugg, M. D. (2001). Electrophysiological correlates of the retrieval of emotional and non-emotional context. Journal of Cognitive Neuroscience, 13, 877891. doi: $10.1162 / 089892901753165809$

Moberly, N. J., \& Watkins, E. R. (2008). Ruminative self-focus and negative affect: an experience sampling study. Journal of Abnormal Psychology, 117, 314-323. doi: 10.1037/0021-843X.117.2.314

Nolen-Hoeksema, S. (2000). The role of rumination in depressive disorders and mixed anxiety/depressive symptoms. Journal of Abnormal Psychology, 109, 504-511. doi:10.1037/0021-843X.109.3.504

Nolen-Hoeksema, S., Larson, J., \& Grayson, C. (1999). Explaining the gender difference in depressive symptoms. Journal of Personality and Social Psychology, 77, 1061-1072. doi: $10.1037 / 0022-3514.77 .5 .1061$ 
Nolen-Hoeksema, S., \& Morrow, J. (1991). A prospective study of depression and posttraumatic stress symptoms after a natural disaster: the 1989 Loma Prieta earthquake. Journal of Personality and Social Psychology, 61, 115-121.doi: $10.1037 / 0022-3514.61 .1 .115$

Nolen-Hoeksema, S., Wisco, B. E., \& Lyubomirsky, S. (2008). Rethinking rumination. Perspectives on Psychological Science, 3, 400-424. doi: 10.1111/j.17456924.2008.00088.x

Peirce, J.W. (2007) PsychoPy - Psychophysics software in Python. Journal of Neuroscience Methods, 162. 8-13. doi: 10.1016/j.jneumeth.2006.11.017

Philippot, P., \& Brutoux, F. (2008). Induced rumination dampens executive processes in dysphoric young adults. Journal of Behavior Therapy and Experimental Psychiatry, 39, 219-227. doi:10.1016/j.jbtep.2007.07.001

Roberts, J. E., Gilboa, E., \& Gotlib, I. H. (1998). Ruminative response style and vulnerability to episodes of dysphoria: Gender, neuroticism, and episode duration. Cognitive Therapy and Research, 22, 401-423. doi: 10.1023/A:1018713313894

Roberts, H., Watkins, E. R., \& Wills. (2013). Cueing an unresolved personal goal causes persistent ruminative self-focus: An experimental evaluation of control theories of rumination. Journal of Behavior Therapy and Experimental Psychology, 44, 449-455. doi:10.1016/j.jbtep.2013.05.004

Rude, S. S., Hertel, P. T., Jarrold, W., Covich, J., \& Hedlund, S. (1999). Depression-related impairments in prospective memory. Cognition \& Emotion, 13, 267-276. doi:10.1080/026999399379276 
Rummel, J., Hepp, J., Klein, S. A., \& Silberleitner, N. (2012). Affective state and event-based prospective memory. Cognition \& Emotion, 26, 351-361. doi:10.1080/02699931.2011.574873

Treynor, W., Gonzalez, R., \& Nolen-Hoeksema, S., (2003). Rumination Reconsidered: A Psychometric Analysis. Cognitive Therapy \& Research, 27, 247-259. doi: $\underline{10.1023 / \mathrm{A}: 1023910315561}$

Watkins, E., \& Brown, R. G. (2002). Rumination and executive function in depression: an experimental study. Journal of Neurology, Neurosurgery \& Psychiatry, 72, 400-402. doi:10.1136/jnnp.72.3.400

Watkins, E., \& Teasdale, J. D. (2001). Rumination and overgeneral memory in depression: Effects of self-focus and analytic thinking. Journal of Abnormal Psychology, 110, 353-357. doi: 10.1037//0021-843X.110.2.333

Watson, P. (2016). Rules of thumb on magnitudes of effect sizes [Webpage]. Retrieved from http://imaging.mrc-cbu.cam.ac.uk/statswiki/FAQ/effectSize

Whitmer, A. J., \& Banich, M. T. (2007). Inhibition versus switching deficits in different forms of rumination. Psychological Science, 18, 546-553. doi:10.1111/j.14679280.2007.01936.x 